\title{
A General Algorithm for Biorthogonal Functions and Performance Analysis of Biorthogonal Scramble Modulation System
}

\author{
Yueyun Chen ${ }^{1,2}$, Zhenhui Tan ${ }^{1}$ \\ ${ }^{1}$ State Key Laboratory of Rail Traffic Control and Safety, Beijing Jiaotong University, Beijing, China \\ ${ }^{2}$ Beijing Science and Technology University, Beijing, China \\ E-mail: chenyy2@sina.com \\ Received August 26, 2009; revised September 7, 2009; accepted November 6, 2009
}

\begin{abstract}
Applying the theorems of Mobius inverse and Dirichlet inverse, a general algorithm to obtain biorthogonal functions based on generalized Fourier series analysis is introduced. In the algorithm, the orthogonal function can be not only Fourier or Legendre series, but also can be any one of all orthogonal function systems. These kinds of biorthogonal function sets are used as scramble signals to construct biorthogonal scramble modulation (BOSM) wireless transmission systems. In a BOSM system, the transmitted signal has significant security performance. Several different BOSM and orthogonal systems are compared on aspects of BER performance and spectrum efficiency, simulation results show that the BOSM systems based on Chebyshev polynomial and Legendre polynomial are better than BOSM system based on Fourier series, also better than orthogonal MCM and OFDM systems.
\end{abstract}

Keywords: Biorthogonal Functions, Biorthogonal Scramble Modulation, Generalized, Fourier Series, Mobius Inverse, Wireless Transmission

\section{Introduction}

In wireless transmission systems, orthogonal signals are often used for transmission information. In order to reduce the complexity of receivers in orthogonal transmission system and improve the bandwidth efficiency, a kind of special biorthogonal modulation was adopted. The biorthogonal signals are consisted of two groups of orthogonal signals [1,2]. In group one, the $\mathrm{M} / 2$ functions are directly obtained from orthogonal function system; the $M / 2$ functions in the other group are the inverse functions of group one's. Therefore, only half of the bandwidth and half correlators were required compared with $\mathrm{M}$ orthogonal functions modulation system. Biorthogonal CDMA is also helpful to improve spectrum efficiency, reduce multiple-access interference and complexity in receiver [3,4]. However, these systems also belong to orthogonal systems because all the biorthogonal signals are also orthogonal.

In fact, security is difficult to be guaranteed in orthogonal systems, because the signals used in modulation and de-modulation are same in transmitter and receiver (sometimes except the symbol "+" or "-"). Recently, a kind of biorthogonal functions which are no-orthogonal was discussed. In paper [5,6], Wei Yuchuan and Chen Nianxian analyzed several periodic waves based on Fourier series expansions with the theorem of Möbius inverse and the extended Möbius inverse theorem proposed by Chen Nanxian [7], pointed out that a periodic signal is able to be represented as the a superposition of some easily generated periodic functions (such as sawtooth wave and triangular wave) with different frequencies, and gave an algorithm to obtain the biorthogonal functions based on Fourier series analysis. The kind of biorthogonal functions is not orthogonal. However, the condition above is that the Fourier coefficients of the period functions are completely multiplicative. Subsequently, $\mathrm{Su}$ Wuxun in paper [8] gave similarly the inverse transform of some often-used symmetrical periodic waveforms based on Fourier series analysis, and suggested a digital communication system architecture using biorthogonal functions as multi-carriers in paper [9]. However, the condition of completely multiplicative Fourier coefficients limited the space of biorthogonal functions.

In paper [10], we introduced an algorithm to obtain biorthogonal functions based on Fourier-Legendre series 
analysis, and proposed a wireless transmission system of biorthogonal scramble modulation (BOSM) based on such kind of biorthogonal function sets. It was pointed out that in flat fading channel, the BOSM systems based on Fourier series analysis have the close BER performance, and spectrum efficiency is similar to MCM system; but the system based on Fourier-Legendre series analysis has higher spectrum efficiency better BER performance than other systems.

In this paper, we introduce a universal algorithm to obtain biorthogonal functions based on General Fourier series analysis. The algorithm doesn't need the condition that the series coefficients are completely multiplicative. In the meantime, the orthogonal function can be not only Fourier or Legendre series, but also can be any one of all orthogonal function systems. Then we propose a BOSM (biorthogonal scramble modulation) system using some of these new biorthogonal functions. On the condition of flat fading channel, we analyze and compare the BER performance and spectrum efficiency of different BOSM and orthogonal systems. The simulating results show that the BOSM based on General Fourier series analysis not only has better BER performance, but also has higher spectrum efficiency than the BOSM system based on Fourier series analysis.

\section{The Fundamental Theorems of Möbius Inverse Transform and Dirichlet Inverse}

Möbius inverse transform and Dirichlet inversion are helpful theorems. The related theorems are briefly introduced in this section.

A basic Möbius inverse formula is described as follows. If $f(n)$ is a number-theoretic function and

$$
F(n)=\sum_{d \mid n} f(d)
$$

then, for all positive integers $n$, there exists

$$
f(n)=\sum_{d \mid n} \mu(d) F\left(\frac{n}{d}\right)
$$

Inversely, if Equation (2) exists, then Equation (1) can be yielded. In above equation, $\mu(n)$ is a Mobius function defined as

$$
\mu(n)=\left\{\begin{array}{cc}
1, & n=1 \\
(-1)^{r} & \text { if all the primes of } n \text { are different } \\
0, & \text { if } n \text { has a squared factor }
\end{array}\right.
$$

If $f(\mathrm{n})$ and $g(n)$ are number-theoretic functions, then their Dirichlet multiplication $h(n)$ is also a numbertheoretic functions [11]

$$
h(n)=\sum_{d \mid n} f(n) g\left(\frac{n}{d}\right)(n=1,2,3, \cdots)
$$

and it can be written as

$$
h(n)=f(n) * g(n)
$$

If $f(1) \neq 0$, there exists a unique number-theoretic function $f^{-1}(n)$ satisfying

$$
f(n) * f^{-1}(n)=f^{-1}(n) * f(n)=\delta_{n-1}
$$

where $\delta_{n-1}$ is Kroneche's delta symbol and $f^{-1}(n)$ is called Dirichlet inverse of $f(n) . f^{-1}(n)$ can be calculated by recurrence formula as following

$$
\begin{aligned}
& f^{-1}(n)=\frac{1}{f(1)}(\mathrm{n}=1) \\
& f^{-1}(n)=\frac{1}{f(1)} \sum_{\substack{d \mid n \\
d<n}} f\left(\frac{n}{d}\right) f^{-1}(d) \quad(n>1)
\end{aligned}
$$

Specially, if $f(x)$ is complete multiplicative, then

$$
f^{-1}(n)=\mu(n) f(n)
$$

Following is a useful Mobius inverse formula. It is expressed as follows $[8,12]$

$$
G(x)=\sum_{n=1}^{\infty} r(n) g(n x) \Leftrightarrow g(x)=\sum_{n=1}^{\infty} r^{-1}(n) G(n x)(8)
$$

where $G(x)$ and $g(x)$ are two functions defined in $(-\infty, \infty)$ and $r^{-1}(d)$ is Dirichlet inverse of $r(d)$.

\section{Fourier Series Analysis}

Function $f(x)$ belongs to function space $L^{2}[-\pi, \pi]$ with period $2 \pi$. It has a unique Fourier series expressed as

$$
f(t)=a(0)+\sum_{n=1}^{\infty}[a(n) \cos (n t)+b(n) \sin (n t)]
$$

where, $a(0), a(n)$ and $b(n)$ are the Fourier series coefficients of $f(t)$.

Supposing that $u(t)$ and $v(t)$ are respectively an even and odd functions with period of $2 \pi$, their Fourier series is expressed respectively as

$$
\begin{aligned}
& u(t)=\sum_{n=1}^{\infty} A(n) \cos (n \omega t) \\
& v(t)=\sum_{n=1}^{\infty} B(n) \sin (n \omega t)
\end{aligned}
$$

By the theorem of Möbius inverse [12], Equation (9) can be rewritten as the following form 


$$
\begin{aligned}
f(t) & =a(0)+\sum_{n=1}^{\infty}\left[a(n) \sum_{m=1}^{\infty} A^{-1}(m) u(m n t)\right]+\sum_{n=1}^{\infty}\left[b(n) \sum_{m=1}^{\infty} B^{-1}(m) v(m n t)\right] \\
& =a(0)+\sum_{k=1}^{\infty} \sum_{d \mid k}^{\infty} a(d) A^{-1}\left(\frac{k}{d}\right) u(k t)+\sum_{k=1}^{\infty} \sum_{d \mid k}^{\infty} b(d) B^{-1}\left(\frac{k}{d}\right) v(k t) \\
& =a(0)+\sum_{k=1}^{\infty} c(k) u(k t)+\sum_{k=1}^{\infty} d(k) v(k t)
\end{aligned}
$$

where

$$
\begin{aligned}
c(k) & =\sum_{d \mid k}^{\infty} a(d) A^{-1}\left(\frac{k}{d}\right) \\
& =\sum_{d \mid k}^{\infty} \frac{1}{\pi} \int_{-\pi}^{\pi} f(t) \cos (d t) d t \cdot A^{-1}\left(\frac{k}{d}\right) \\
& =\int_{-\pi}^{\pi} f(t) g_{k}(t) d t \\
d(k) & =\sum_{d \mid k}^{\infty} b(d) B^{-1}\left(\frac{k}{d}\right) \\
& =\sum_{d \mid k}^{\infty} \frac{1}{\pi} \int_{-\pi}^{\pi} f(t) \sin (d t) d t \cdot B^{-1}\left(\frac{k}{d}\right) \\
& =\int_{-\pi}^{\pi} f(t) h_{k}(t) d t
\end{aligned}
$$

Equation (10) shows that a quadratically integrabel function can be decomposed as the superposition of even function $u(t)$ and $v(t)$ and odd function $v(t)$ with different frequencies. In Equation (11) and Equation (12), $g_{k}(t)$ and $h_{k}(t)$ is given respectively by

$$
\begin{aligned}
& g_{k}(t)=\frac{1}{\pi} \sum_{d \mid k}^{\infty} A^{-1}\left(\frac{k}{d}\right) \cos d t \\
& h_{k}(t)=\frac{1}{\pi} \sum_{d \mid k}^{\infty} B^{-1}\left(\frac{k}{d}\right) \sin d t
\end{aligned}
$$

It can be proved that

$$
\begin{gathered}
\int_{-\pi}^{\pi} u(m t) g_{n}(t) d t=\delta_{n m} \\
\int_{-\pi}^{\pi} v(m t) h_{n}(t) d t=\delta_{n m}
\end{gathered}
$$

namely, $\quad g_{k}(t)$ and $u(m t)=\sum_{n=1}^{\infty} A(n) \cos (m n t)$ are biorthogonal functions, the same are $h_{k}(t)$ and $v(m t)=\sum_{n=1}^{\infty} B(n) \sin (m n t)$. In Equation (10), $u(t)$ and $v(t)$ are called the basic function of biorthogonal functions. In fact, the proof of Equation (15) and Equation (16) do not need the condition that Fourier coefficients $A(n)$ or $B(n)$ of $u(t)$ or $v(t)$ are completely multiplicative. When $A(n)$ or $B(n)$ are not completely multiplicative, i.e., $A^{-1}(n) \neq \mu(n) A(n) \quad$ or
$B^{-1}(n) \neq \mu(n) B(n)$, the conclusion of Equation (15) and Equation (16) can also be obtained.

\section{General Fourier Series Analysis}

Supposing $P_{n}(x)$ is an infinite orthogonal series with weight function $h(x)$ in the orthogonal interval $[a, b]$, namely

$$
\int_{a}^{b} h(x) P_{m}(x) P_{n}(x) d x= \begin{cases}0, & m \neq n \\ 1 & m \neq n\end{cases}
$$

where weight function $h(x)$ is nonnegative and integrable in the interval $[a, b]$ [13]. If $f(x)$ is a function defined in interval $[a, b]$ and satisfies $\int_{a}^{b} h(x) f^{2}(x) d x<\infty$, then it can be decomposed to generalized Fourier series as

$$
f(x)=\sum_{n=0}^{\infty} a(n) P_{n}(x)
$$

The coefficient of generalized Fourier series $a(n)$ is defined by

$$
a(n)=\int_{a}^{b} h(x) f(x) P_{n}(x) d x
$$

Then, a more universal algorithm to obtain biorthogonal functions is introduced by following proposition.

Proposition 1. Supposing $P_{n}(x)$ is orthogonal series with weighted function $h(x)$ in orthogonal interval $[a, b]$. If $f(x)$ is a function defined in interval $[a, b]$ and satisfies the relation of $\int_{a}^{b} h(x) f^{2}(x) d x<\infty$ (i.e. $f(x)$ belongs to $[a, b ; h(x)])$. Then, the two group functions

$$
F_{m}(x)=\sum_{n=1}^{\infty} a(n) P_{n m}(x), \quad n m \leq K
$$

and

$$
\tilde{F}_{n}(x)=\sum_{d \mid k} a^{-1}\left(\frac{n}{d}\right) h(x) P d(x)
$$

is a biorthogonal function set, namely

$$
\int_{a}^{b} F_{m}(x) \tilde{F}_{n}(x) d x=\delta_{m n}
$$

where $a(n)$ is the coefficient of generalized Fourier series of $f(x), a^{-1}(n)$ is its Dirichlet inverse, and $K$ is the order of biorthogonal function set.

\section{Proof.}




$$
\begin{aligned}
\int_{a}^{b} F_{m}(x) \cdot \tilde{F}_{n}(x) d x & =\int_{a}^{b} \sum_{i=1}^{\infty} a(i) P_{i m}(x) \cdot \sum_{d \mid n} a^{-1}\left(\frac{n}{d}\right) h(x) P d(x) d x \\
& =\sum_{i=1}^{\infty} a(i) \sum_{d \mid n} a^{-1}\left(\frac{n}{d}\right) \cdot \delta_{i m, d} \\
& =\sum_{\substack{d|n \\
m| n}} a\left(\frac{d}{m}\right) a^{-1}\left(\frac{n}{d}\right) \\
& =\sum_{\substack{d|n \\
m| n}} a\left(\frac{d}{m}\right) a^{-1}\left(\frac{n / m}{d / m}\right)=\delta_{m n}
\end{aligned}
$$

The proof is completed.

In Proposition $1, P_{n}(x)$ represents any a kind of orthogonal series expanded in orthogonal function system $\left\{P_{n}(x)\right\}$. With the difference of $h(x), P_{n}(x)$ can be different orthogonal polynomials series, such as Legendre polynomials, Chabyshev polynomials, Hermite polynomials, Laguarre polynomials, etc. The particular case is Fourier series when $h(x)=1$ and $P_{n}(x)$ is trigonometric function. Therefore, the algorithm given by Equation (20) and Equation (21) is a general algorithm to obtain biorthogonal functions. In fact, the process of biorthogonalizing corresponds to rotate the orthogonal polynomials coordinate axis, thus one function in bi-orthogonal function set has projection on one or more axis.

There is a fast algorithm for biorthogonal functions. Functions (20) and (21) can be rewritten as matrix forms as

$$
\begin{aligned}
F^{\prime} & =\left[\begin{array}{llllllll}
F_{1}^{\prime}(x) & F_{2}^{\prime}(x) & F_{3}^{\prime}(x) & F_{4}^{\prime}(x) & \cdots & F_{K}^{\prime}(x)
\end{array}\right]^{T} \\
& =\left[\begin{array}{cccccccc}
a(1) & a(2) & a(3) & a(4) & a(5) & a(6) & \cdots & a(K) \\
0 & a(1) & 0 & a(2) & 0 & a(3) & \cdots & \\
0 & 0 & a(1) & 0 & 0 & a(2) & \cdots & \\
0 & 0 & 0 & a(1) & 0 & 0 & \cdots & \\
\vdots & \vdots & \vdots & \vdots & \vdots & \vdots & \vdots & \\
0 & 0 & 0 & 0 & 0 & & \cdots & a(1)
\end{array}\right]\left[\begin{array}{c}
P_{1}(x) \\
P_{2}(x) \\
P_{3}(x) \\
P_{4}(x) \\
\vdots \\
P_{K}(x)
\end{array}\right] \\
& =A^{\prime} P(x)
\end{aligned}
$$

$$
\begin{aligned}
& \tilde{F}^{\prime}=\left[\begin{array}{llllll}
\tilde{F}_{1}^{\prime}(x) & \tilde{F}_{2}^{\prime}(x) & \tilde{F}_{3}^{\prime}(x) & \tilde{F}_{4}^{\prime}(x) & \cdots & \tilde{F}_{K}^{\prime}(x)
\end{array}\right]^{T} \\
& =h(x)\left[\begin{array}{cccccc}
a^{-1}(1) & 0 & 0 & 0 & \cdots & 0 \\
a^{-1}(2) & a^{-1}(1) & 0 & 0 & \cdots & 0 \\
a^{-1}(3) & 0 & a^{-1}(1) & 0 & \cdots & 0 \\
a^{-1}(4) & a^{-1}(2) & 0 & a^{-1}(1) & \cdots & 0 \\
a^{-1}(5) & 0 & 0 & 0 & \cdots & 0 \\
a^{-1}(6) & a^{-1}(3) & a^{-1}(2) & 0 & \cdots & 0 \\
\vdots & \vdots & \vdots & \vdots & \vdots & 0 \\
a^{-1}(K) & \cdots & \cdots & \cdots & \cdots & a^{-1}(1)
\end{array}\right]\left[\begin{array}{c}
P_{1}(x) \\
P_{2}(x) \\
P_{3}(x) \\
P_{4}(x) \\
\vdots \\
P_{K}(x)
\end{array}\right] \\
& =h(x) B^{\prime} P(x)
\end{aligned}
$$

where $K$ is called the order of biorthogonal function set. If matrix $A$ and $B$ is normalized individually by $a(1)$ and $a^{-1}(1)$, Equation (23) and Equation (24) are rewritten as

$$
\begin{aligned}
& F=A P(x) \\
& \tilde{F}=h(x) B P(x)
\end{aligned}
$$

where $A=A^{\prime} / a(1), B=B^{\prime} / a^{-1}(1)$. Then, matrix $A$ and $B$ are sparse triangular matrixes and they are transposes each other (regardless the upper-mark "-1"). Therefore, the calculation speed of the algorithm method is improved rapidly.

Following takes Chabyshev polynomials as an example. Chabyshev polynomials is defined in orthogonal interval $[-1,1]$ with weight function $h(x)=1 / \sqrt{1-x^{2}}$. The series can be expressed as

$$
T_{n}(x)=\cos (n \arccos x) \quad(n=0,1,2, \cdots)
$$

If $f(x)=\ln (1+x)=-\ln 2+2 \sum_{n=1}^{\infty} \frac{(-1)^{n}}{n} T_{n}(x)$, then $a(n)=2(-1)^{n-1} / n,(n \geq 1)$, and $a(n)^{-1}$ can be calculated by Equation (6) and Equation (7). Let $K=6$, then the biorthogonal functions can be directly obtained as follows

$$
\left[\begin{array}{l}
F_{1}(x) \\
F_{2}(x) \\
F_{3}(x) \\
F_{4}(x) \\
F_{5}(x) \\
F_{6}(x)
\end{array}\right]=\left[\begin{array}{cccccc}
1 & -1 / 2 & 1 / 3 & -1 / 4 & 1 / 5 & -1 / 6 \\
0 & 1 & 0 & -1 / 2 & 0 & -1 / 4 \\
0 & 0 & 1 & 0 & 0 & -1 / 2 \\
0 & 0 & 0 & 1 & 0 & 0 \\
0 & 0 & 0 & 0 & 1 & 0 \\
0 & 0 & 0 & 0 & 0 & 1
\end{array}\right]=\left[\begin{array}{c}
P_{1}(x) \\
P_{2}(x) \\
P_{3}(x) \\
P_{4}(x) \\
P_{5}(x) \\
P_{6}(x)
\end{array}\right]
$$

$$
\left[\begin{array}{c}
\tilde{F}_{1}(x) \\
\tilde{F}_{2}(x) \\
\tilde{F}_{3}(x) \\
\tilde{F}_{4}(x) \\
\tilde{F}_{5}(x) \\
\tilde{F}_{6}(x)
\end{array}\right]=\frac{1}{\sqrt{1-x^{2}}}\left[\begin{array}{cccccc}
1 & 0 & 0 & 0 & 0 & 0 \\
1 / 2 & 1 & 0 & 0 & 0 & 0 \\
-1 / 3 & 0 & 1 & 0 & 0 & 0 \\
1 / 2 & 1 / 2 & 0 & 1 & 0 & 0 \\
-1 / 5 & 0 & 0 & 0 & 1 & 0 \\
-1 / 6 & -1 / 3 & 1 / 2 & 0 & 0 & 1
\end{array}\right]=\left[\begin{array}{c}
P_{1}(x) \\
P_{2}(x) \\
P_{3}(x) \\
P_{4}(x) \\
P_{5}(x) \\
P_{6}(x)
\end{array}\right]
$$

It can be proved that functions $\left\{F_{m}(x)\right\}$ and $\left\{\tilde{F}_{m}(x)\right\}$ are biorthogonal, and the functions in one group are not orthogonal. So, this is a kind of more general biorthogonal functions.

\section{Transmission System of BOSM}

The biorthogonal function sets discussed above have a magnetic feature that the functions in same one group are 
not orthogonal in time and frequency region. If the functions of one group are mixed up, it is very difficult to apart each one of them from the mixture if without the other group of biorthogonal functions. Profiting from this feature, a wireless security transmission system based this kind of comprehensive biorthogonal function can be constructed. Using the biorthogonal function sets as scramble modulation signal, the systems have outstanding security performance. We call the system as Bi-Orthogonal Scrambling Modulation (BOSM) transmission system. The principle diagram is shown in Figure 2.

The input sequence $d_{i}$ is converted to $K$ parallel sub-sequences $d_{p i}(1 \leq i \leq K)$ which is individually scrambled by the biorthogonal signal $\left\{F_{1}(x), F_{2}(x), \cdots, F_{K}(x)\right\}$ called BOSS (biorthogonal scramble signal) and the variable $x$ is treated as time $t$. The transmitted symbol $s(t)$ is performed by mixing all the outputs of $K$ parallel branches. It is expressed as

$$
s(t)=\sum_{i=1}^{K} d_{p i}(t) F_{i}(t)
$$

Supposing that the mixed signal $s(t)$ is transmitted over flat fading channel and the transmitted signal is compensated effectively in receiver. Then, the received signal $r(t)$ is expressed as

$$
r(t)=s(t)+n(t)=\sum_{i=1}^{K} d_{p i}(t) F_{i}(t)+n(t)
$$

where $n(t)$ is AWGN with mean $=0$, variance $\sigma_{0}^{2}$. Further, supposing that the scramble signal in receiver has been already synchronized with transmitter's. The received signal $r(t)$ is correlated individually by $\left\{\tilde{F}_{1}(t), \tilde{F}_{2}(t), \cdots, \tilde{F}_{L}(t)\right\}$, and the output of the $j$-th correlator is

$$
\begin{aligned}
d_{p i}^{\prime} & =\int_{0}^{T} r(t) \tilde{F}_{j}(t) d t=\sum_{i=1}^{K} d_{p i}(t) \int_{0}^{T} F_{i}(t) \tilde{F}_{j}(t) d t+\int_{0}^{T} n(t) \tilde{F}_{j}(t) d t \\
& =\left\{\begin{array}{cl}
\alpha_{i} d_{p i}(t) & i=j \\
n^{\prime}(t) & i \neq j
\end{array}\right.
\end{aligned}
$$

where $\alpha_{i}$ is a constant. When the correlating process is finished, the de-scramble process is completed also.

\section{Simulation Analysis for Transmission Performance of BOSM System}

In this section, we discuss BER performance, spectrum efficiency and the impact of synchronization precision in BOSM system. The simulation system is established according to Figure 1. Considering the scene of flat fad- ing channel in narrow band condition, let $K=6$ and symbol rate $R_{s}=4800 \mathrm{baud} / \mathrm{s}$. In receiver, the decision criterion is ML (maximum likelihood). Because parallel transmission depresses ISI (inter-symbol interference) remarkably, the BER performance is mainly affected by channel and ICI (inter-channel interference).

We compare different BOSM and orthogonal systems on the performance of BER and spectrum efficiency. They are based on different BOSS which are obtained respectively by Chebyshev polynomial, Legendre polynomial (general Fourier series) and Fourier series analysis.

The basic function for Chebyshev polynomial analysis is the example given in Section 4. For Legendre polynomial analysis, the basic function is as following

$$
f(x)=\left\{\begin{array}{cc}
1, & 0<x \leq 1 \\
-1, & -1 \leq x<0
\end{array}\right.
$$

An even symmetrical trapezoid is used as the basic function for Fourier series analysis, expressed as

$$
\operatorname{Tra}(t)=\left\{\begin{array}{cc}
t+\pi & -\pi \leq t \leq-\pi / 2 \\
\pi / 2 & -\pi / 2 \leq t \leq \pi / 2 \\
-t+\pi & \pi / 2 \leq t \leq \pi
\end{array}\right.
$$

Correspondingly, the obtained biorthogonal functions sets are marked as BOSS-C, BOSS-L, and BOSS-F, individually. Figure 2 shows the transmitted signal waveform of the example of BOSS-C. It seems like noise.

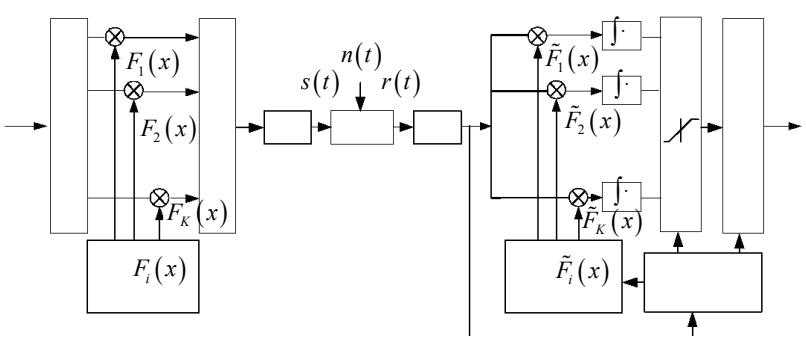

Figure 1. Block diagram of baseband BOSM system.

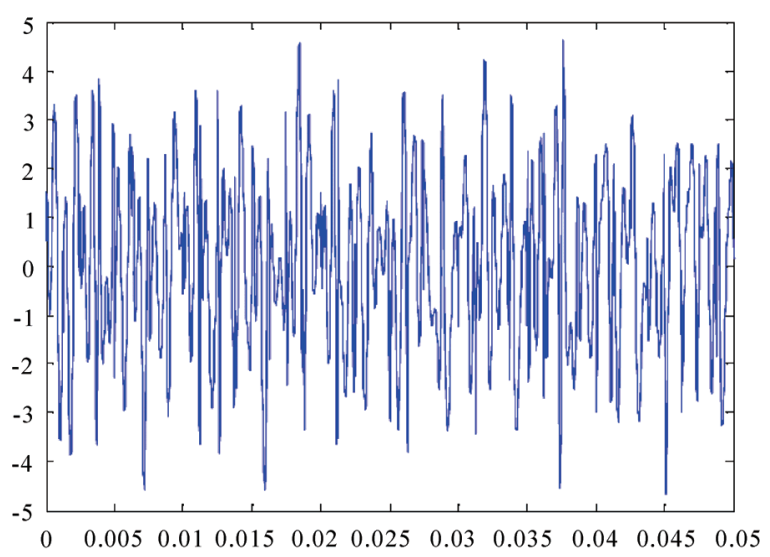

Figure 2. Waveform of mixed signal. 


\subsection{Spectrum Efficiency}

In BOSS-F, the bandwidth of mixed signal is $B \approx 14 \mathrm{kHz}$ (see Figure 3), the spectrum efficiency is $0.34 \mathrm{baud} / \mathrm{Hz}$ approximately, and equal to orthogonal MCM (Multiple Carriers Modulation) system regardless the guard band.

In BOSS-C and BOSS-L, the PSD (power spectrum density) of mixed signal is shown respectively in Figures 4(a) and 4(b). The energy of mixed signal is mainly concentrated in interval of $0 \sim 3.2 \mathrm{kHz}$ and $0 \sim 4.8 \mathrm{kHz}$, and the

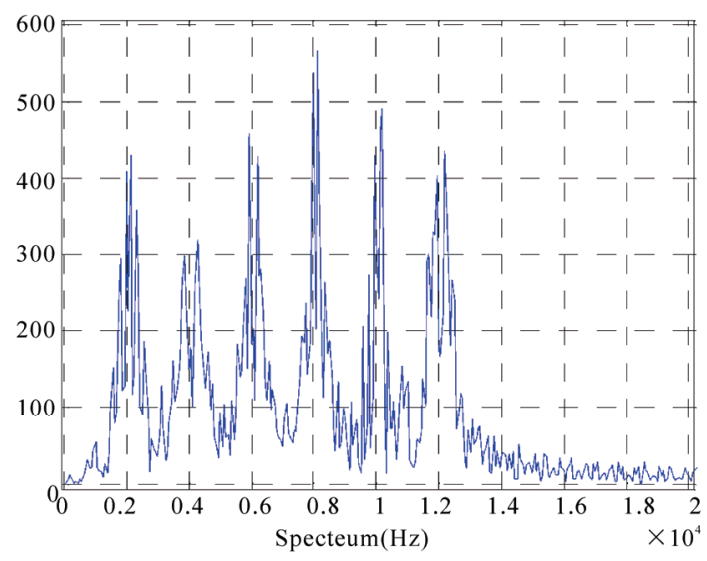

Figure 3. Spectrum of mixed signal for BOSS-F.

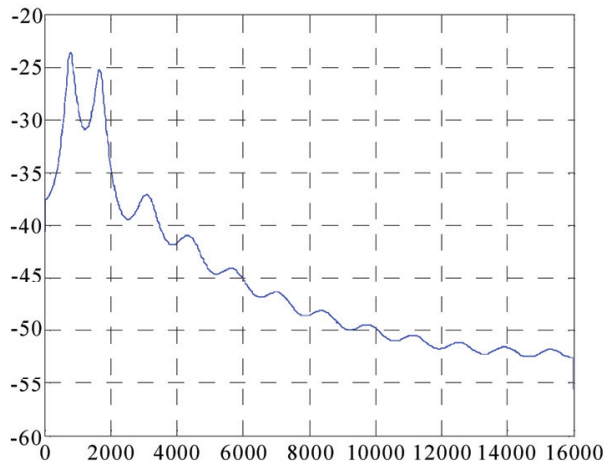

(a)

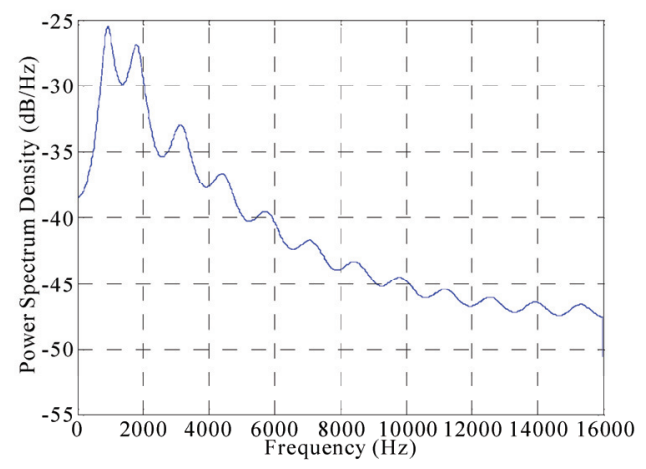

(b)

Figure 4. Single-side PSD of mixed signal. (a) BOSS-C; (b) BOSS-L. spectrum efficiency approximately is $1 \mathrm{baud} / \mathrm{Hz}$ and 1.5 baud $/ \mathrm{Hz}$ respectively. In fact, the PSD of mixed signals are changed a bit with the change of basic function $f(t)$.

In OFDM system, the spectrum efficiency is improved approaching 1 times than orthogonal MCM system. Compared with OFDM system, the BOSM system based on 10 Legendre polynomial analyses has similar performance of spectrum efficiency. Therefore, the BOSM systems based on general Fourier series analysis, including Chebyshev series and Legendre series analysis, have higher spectrum efficiency.

\subsection{BER Performance}

In paper [10], we had given a conclusion that the BOSM systems based on Fourier series with different basic functions have close BER performance. Supposing synchronization is exactly established. The BER performance of different systems is shown in Figure 5. It reveals that, in flat fading channel, the BER performance of BOSS-L and BOSS-C systems are the best, and BOSS-F and orthogonal-M systems (or OFDM system) have the close BER performance. Therefore, the BER performance of the BOSM systems based on general Fourier series analysis is satisfying.

\subsection{Synchronization Property}

Synchronization error of biorthogonal signals between transmitter and receiver leads to ICI. Supposing the error is $\Delta \tau$, then, the output of $j$-th correlator is $(n(t)$ is omitted)

$$
\begin{aligned}
d_{p i}^{\prime} & =\sum_{i=1}^{K} d_{p i}(t) \int_{0}^{T} F_{i}(t) \tilde{F}_{j}(t+\Delta \tau) d t \\
& =\sum_{i=1}^{K} d_{p i}(t) \int_{0}^{T} \sum_{k=1}^{K} A_{i k} P_{i} \sum_{l=1}^{K} B_{j l} P_{j}(t+\Delta \tau) d t \\
& =\sum_{i=1}^{K} d_{p i}(t) \sum_{k=1}^{K} \sum_{l=1}^{K} A_{i k} B_{j l} \int_{0}^{T} P_{i}(t) P_{j}(t+\Delta \tau) d t
\end{aligned}
$$

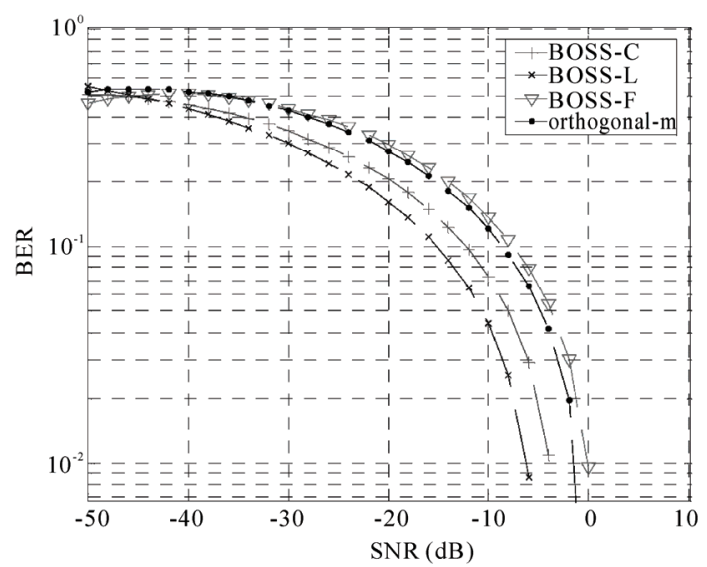

Figure 5. Spectrum of mixed signal. 
In above expression, the output is impacted by all other K-1 channels, because the biorthogonal feature is damaged in different degree with different $\Delta \tau$. When using the example of BOSS-C, Figure 6 shows the impact of synchronization error to BER performance. The BER curve is changed periodically with the synchronization error, and the period is the length of interval [a,b] (sample number 40 represents the interval length). Therefore, synchronization precision needed to be guarantied in BOSM system, just same as OFDM systems.

\section{Conclusions}

The biorthogonal functions were introduced into ordinary orthogonal function systems, and pointed out that the condition of complete multiplication is not needed. In the meantime, a more general algorithm for biorthogonal functions called general Fourier series analysis was proposed. Because the obtained biorthogonal functions are not orthogonal each other, the BOSS that the obtained biorthogonal functions were used as scramble signal to be modulated by transmitted symbols has outstanding security performance, especially when the order is enough big. This is very different from orthogonal system and traditional biorthogonal modulation system. By simulation analysis, in flat fading channel, the BOSM systems based on general Fourier series analysis, such as Chebyshev polynomial and Legendre polynomial analysis, have better BER performance and spectrum efficiency than the BOSM system based on Fourier series analysis, orthogonal MCM system or OFDM system.

However, the demand of high synchronization precision and having high PAPR (peak average power rate) in BOSM systems are two primary problems, just as OFDM or multi-carriers systems, and the degree of PAPR rises with increasing of the order of BOSS. Because smaller amplitude of polynomial coefficients in biorthogonal functions appears with the increasing of the order of biorthogonal function set, the performance of demodulation

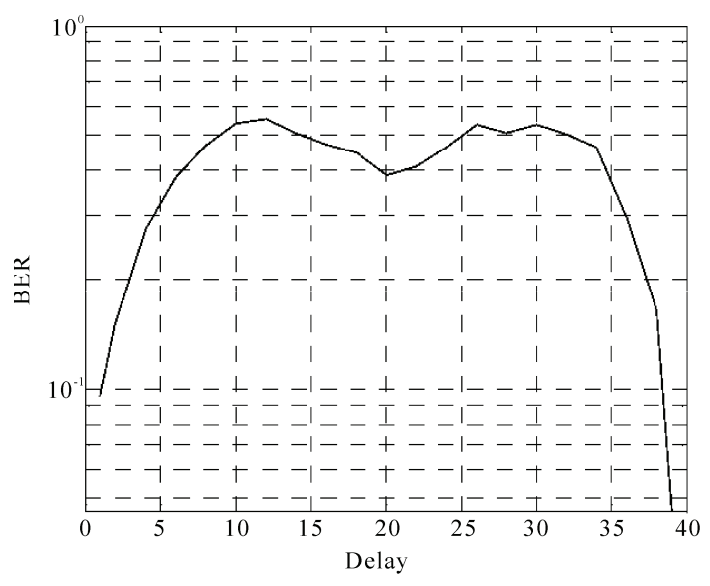

Figure 6. Impact on BER by synchronization error. error probability will be deteriorate when the energy in a symbol interval is significantly weaker than the considered noise level. Therefore, the order $K$ should be not too big.

To properly choose the basic function can change the PDF and PAPR of transmitted signal. In order to make BOSM systems with better performance, how to construct a proper basic function in an orthogonal function system to obtain the biorthogonal functions, it is still a challenge.

\section{References}

[1] R. B. Blizard, "Comparison of biorthogonal and bisimplex codes," IEEE Transactions on Communications Technology, Vol. 15, No. 4, pp. 657-658, August 1967.

[2] M. Kim and S. Tretter, "Performance of sequential decoding with biorthogonal modulation and Q-level quantization," IEEE Transactions on Communications, Vol. 19, pp. 88-92, February 1971.

[3] S.-H. Hong and J.-S. No, "Performance analysis of CDMA systems by using biorthogonal codes," IEEE 45th Conference on Vehicular Technology, Vol. 2, pp. 694698, July 1995.

[4] S.-J. Kang, D.-K. Hong, et al., "Constant-amplitude multicode-biorthogonal modulation," IEEE Transactions on Communications, Vol. 55, No. 1, pp. 69-75, January 2007.

[5] Y. Wei and N. Chen, "Square wave analysis," Journal of Mathematical Physics, Vol. 39, No. 8, pp. 4226-4245, August 1998.

[6] Y. Wei, "Dirichlet multiplication and easily-generated function analysis," Computers \& Mathematical with Applications, Vol. 39, pp. 173-199, 2000.

[7] N. Chen, "Modified Möbius inverse formula and its applications in physics," Physics Review Letters, Vol. 64, No. 11, pp. 1193-1195, 1990.

[8] W. Su, W. Zhang, and J. Wang, "The evaluations of the inverse transform of eight often-used waveforms by Möbius transform-The inverse transform of their Fourier series," Chinese of Journal Electronics, Vol. 14, No. 3, pp. 513-518, July 2005.

[9] C. Ling, F. Chen, and W. Su, "The Chen-mobius multi-carriers digital communication system and its simulation," IEEE International Workshop on Anti-Counterfeiting, Security and Identification, pp. 16-18, April 2007.

[10] Y. Chen, J. Zhang, and Z. Tan, "A kind of biorthogonal functions based on Fourier-Legendre series and its application in wireless transmission system," IEEE 9th International Conference on Signal Processing, 2008.

[11] K. H. Rosen, "Elementary number theory and its application," 5th edition, Pearson Education Inc. Press, 2005.

[12] M. R. Schroeder, "Number theory in science and communication," 4th edition, Springer-Verlag Press, New York, 2006.

[13] Z. Liu, "Orthogonal function and its application," National Defense Industry Publisher, Beijing, 1982. 